\title{
Desigualdad, equidad e inclusión en la educación superior de Honduras
}

\section{Inequality, equity and inclusion in higher education in Honduras}

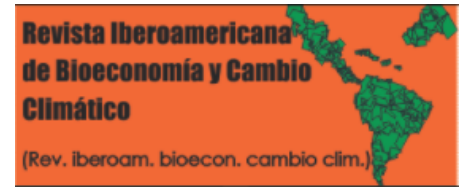

\author{
Mejía Castillo, Harin Joel; Molina Zavala, Ariel; Zuniga, Fran \\ Humberto; Carbajal, Fidel Ángel; Editor Academico Prof. Ph.D Angle \\ Sol-Sanchez
}

\author{
Harin Joel Mejía Castillo \\ harinjoel@post.unanleon.edu.ni \\ Universidad Nacional de Agricultura UNA-Honduras, \\ CUR Somotillo, Honduras \\ Ariel Molina Zavala \\ ariel_zavala55@yahoo.es \\ Universidad Nacional de Agricultura UNA-Honduras, \\ CUR Somotillo, Honduras \\ Fran Humberto Zuniga \\ fran_zunmez@yahoo.com \\ Universidad Nacional de Agricultura UNA-Honduras, \\ CUR Somotillo, Honduras \\ Fidel Ángel Carbajal \\ carbajalfidel@gmail.com \\ Universidad Nacional de Agricultura UNA-Honduras, \\ CUR Somotillo, Honduras \\ Editor Academico Prof. Ph.D Angle Sol-Sanchez \\ sol@colpos.mx \\ Colegio de Postgraduados, Mexico
}

Revista Iberoamericana de Bioeconomía y Cambio Climático

Universidad Nacional Autónoma de Nicaragua, León, Nicaragua ISSN-e: 2410-7980

Periodicidad: Semestral

vol. 3, núm. 5, 2017

czuniga@ct.unanleon.edu.ni

Recepción: 16 Enero 2017

Aprobación: 26 Julio 2017

URL: http://portal.amelica.org/ameli/journal/394/3941752004,

DOI: https://doi.org/10.5377/ribcc.v3i5.5941

Autor de correspondencia: harinjoel@post.unanleon.edu.ni
Resumen: El presente documento constituye un análisis de la situación actual de la educación en Honduras en cuanto a la desigualdad, inclusión y equidad educativa. Para ello es fundamental y necesario conocer las tendencias políticas del país y de la región. Los parámetros evaluados se han vinculado fuertemente con la pobreza generada por las distintas oportunidades en educación, empleo y bajos ingresos económicos. En Honduras se muestra una ligera reducción de la desigualdad en 2011 respecto al 2009 esto a pesar de: la crisis política y social vivida en el país en 2009. Los actuales esfuerzos por mejorar la equidad de la educación se enfocan en la búsqueda de intervenciones que, gestionando los escasos recursos disponibles, disminuyan las brechas en el acceso y el desempeño escolar asociados a las diferencias por estratos socioeconómicos, grupos étnicos, género, o por región de residencia de los estudiantes.

Palabras clave: Inequidad, Poder económico, Grupos étnicos.

Abstract: This document constitutes an analysis of the current situation of education inHonduras in terms of inequality, inclusion and educational equity. For this it is fundamental and It is necessary to know the political tendencies of the country and the region. The evaluated parameters are have strongly linked with the poverty generated by the different opportunities in education, employment and low economic income. In Honduras, there is a slight reduction in the inequality in 2011 compared to 2009 this despite: the political and social crisis experienced in the country in 2009. Current efforts to improve equity in education focus on the search of interventions that, managing the scarce resources available, reduce the gaps in the access and school performance associated with differences by socioeconomic strata, groupsethnicity, gender, or region of student residence.

Keywords: Inequality, Economic power, Ethnic groups.

\section{Notas DE AUTOR}




\section{INTRODUCCIÓN}

Existen factores que determinan la tendencia de las políticas de equidad e inclusión en la educación de la región de américa latina, entre ellos la desigualdad y la exclusión; en ese sentido Aponte (2013) argumenta que "superar la desigualdad y exclusión como estrategia para llegar al camino hacia un desarrollo sustentable de las sociedades, es quizás el reto más apremiante e importante de estos tiempos para los países (pág. 8).

Según Ferreira et al. (2013) las desigualdades que podemos cuantificar se agravan debido a desigualdades cualitativas que también podemos medir y contar con relativa precisión, el número de escuelas y el número de niños matriculados en ellas. Sin embargo, construir más escuelas no es garantía de una educación de calidad. En este panorama, la Alianza Mundial para la Educación (2014) describe que "las escuelas con un cuadro docente de maestros sin preparación profundizan las desigualdades; los vecindarios inseguros y los edificios escolares inseguros vuelven imposible el aprendizaje” (pág. 9).

\section{EDUCACIÓN SUPERIOR Y DESIGUALDAD SOCIAL}

El surgimiento de la Educación superior como tal en la Republica de Honduras surge en el año 1847, es así que durante 130 años el país conto con solamente una institución de educación de ese nivel, hasta el año 1978 que se abren dos nuevos centros universitarios, para la década de los ochenta se apertura cuatro centros, en la década de los noventa seis centros y en la pasada última década siete centros para hacer un total hasta el año 2011 de veinte centros de educación superior (Flores, 2012).

En este devenir histórico, "la educación superior en Honduras ha pasado por diferentes etapas, caracterizadas en alguna manera por un crecimiento sostenido, ordenado y regulado” (Padilla, 2011, pág. 82). Para entender la situación actual de la educación superior en el país, es importante y necesario repasar de manera sintética el desarrollo histórico de este nivel educativo, identificando en dicha historia las lecciones aprendidas y las bases para una mejora del sistema y sus instituciones, a la vez que generar las evidencias sobre lo que ha significado el papel del estado hondureño en este tema (Padilla, 2011).

Sin embargo, al día de hoy en Honduras sigue siendo el segundo país de Centroamérica con menor cantidad de Universidades, superada sólo por Guatemala. En Honduras existen 388 carreras autorizadas, de las cuales 58 son a nivel de grado asociado (técnicos universitarios), 205 licenciaturas, 93 maestrías, 27 especialidades y sub-especialidades y 5 doctorados. Con estos números, la cobertura de la educación superior ronda el 15\% (Duriez, López, \& Moncada, 2016).

Estos datos nos reflejan las brechas sociales que marcan profundamente el contexto en que se desempeña el sistema educativo hondureño. Honduras presenta una de las mayores desigualdades sociales a nivel regional y mundial. En el año 2004, el PNUD en su Informe sobre Desarrollo Humano a nivel mundial ubicaba a Honduras en el lugar $105^{\circ}$ de 121 posibles en términos de desigualdad social, de forma análoga, la CEPAL en su Panorama Social de América Latina 2009, ubica a Honduras en el último lugar de la región al comparar los ingresos entre el quintil más rico y el más pobre (Alas \& Moncada, 2011).

Uno de los factores que influye en los niveles de desigualdad educativa es el funcionamiento del sistema educativo, "esto implica la asignación de los recursos financieros, los criterios de elegibilidad de los programas de asistencia, las becas entre otros y no más importante la calidad de la educación” (Espino, 2015, pág. 18).

\section{AVANCES EN LA REDUCCIÓN DE LA DESIGUALDAD EDUCATIVA}

Para reducir la desigualdad es necesario incrementar el poder económico de un país y que este pueda realizar inversiones en acceso a la educación; en este sentido, es "claro que la igualdad de acceso no es suficiente para garantizar la equidad educativa si no se proporcionan programas de similar calidad a todas 
las personas, apoyándoles para lograr aprendizajes semejantes, sea cual fuere su condición social, cultural e individual" (Solis \& Moncada, 2016, pág. 137).

En la actualidad se conoce que los "estudiantes con peores condiciones socioeconómicas tienen menos oportunidades y acceso a educación que les provea las técnicas y conocimientos para encontrar empleo remunerado y participar plenamente en los beneficios que la sociedad en la que viven genera" (RodriguezMontano, 2013, pág. 161). En los últimos años, "la cobertura de acceso en la educación superior en Honduras presenta una tendencia positiva, sin embargo, dista de alcanzar los estándares internacionales establecidos para dejar de ser una oportunidad de minorías" (Duriez, López, \& Moncada, 2016, pág. 15).

En Honduras se han realizado varias investigaciones para determinar el nivel de la desigualdad educativa, en un estudio realizado por Espino (2015), los resultados muestran avances importantes en los últimos años:

Una ligera reducción en 2011 respecto al 2009 esto a pesar de: la crisis política y social vivida en el país en 2009, del aumento en la desigualdad de los ingresos y la reducción del gasto público en educación (medido como porcentaje del Producto Interno Bruto-PIB) en 2011 con relación al 2009 (pág. 2).

\section{INCLUSIÓN EDUCATIVA HONDUREÑA}

En un estudio realizado por Paya (2010) describe que "hace ya dos décadas que el concepto de la educación inclusiva apareció en el contexto internacional, en el escenario de la Educación para Todos de la Conferencia Mundial de Jomtien (pág. 127)”. En el transcurso de este tiempo, ha sufrido una evolución positiva, transitando desde la integración relacionada con reformas adicionales para acomodar a alumnos considerados especiales en un sistema escolar tradicional, hasta la actualidad, donde la educación inclusiva conlleva la reestructuración de las escuelas según las necesidades de todos y todas (Rico, 2011).

Uno de los aspectos principales en educación, es tener el acceso a ella en el lugar donde se vive, por ello, Solís \& Moncada (2011) mencionan que:

En Honduras, el área que ha sido la menos favorecida en términos del desarrollo socioeconómico, es el área rural como se mencionó en la caracterización de la población rural. En el sector educativo, el acceso a la educación primaria en el área rural ha mostrado un indicador favorable en cuanto a disponer de escuelas en las comunidades rurales y en la última década (1990-2000), del total de escuelas hondureñas, la mayoría están ubicadas en las zonas rurales (pág. 25).

Lo realmente importante es garantizar el ejercicio de esos derechos en condiciones de igualdad; para ello se debe erradicar toda forma de discriminación directa o indirecta y "es necesario eliminar todas las barreras existentes para el disfrute de los derechos humanos, ya sea mediante la igualdad material o formal, la no discriminación o la acción positiva" (Davila, Naya, \& Asier, 2010, pág. 101).

\section{LA INCLUSIÓN EN LA EDUCACIÓN SUPERIOR DE LOS GRUPOS ÉTNICOS}

Según Solís (2016) "la igualdad de oportunidades en educación implica no sólo igualdad en el acceso, sino y sobretodo, igualdad en la calidad de la educación que se brinda y en los logros de aprendizaje que alcanzan los alumnos" (p.137). En la región de "América Latina, la desigualdad de oportunidades educativas, en función del nivel socioeconómico de los estudiantes, es una de las más agudas en la región, de lo que se deduce que la educación no está siendo capaz de romper el círculo vicioso de la pobreza y la desigualdad social" (Solis \& Godoy, 2016, pág. 139).

Como lo expone Mato (2011) actualmente, las constituciones de la mayoría de los países latinoamericanos reconocen a los pueblos indígenas derechos de idioma, identidad y otros de carácter cultural. Hasta el presente este reconocimiento está consagrado en las constituciones de Argentina, Bolivia, Brasil, Colombia, Costa 
Rica, Ecuador, El Salvador, Guatemala, Honduras, Guyana, México, Nicaragua, Panamá, Paraguay, Perú y Venezuela (pág. 67).

En Honduras la inclusión de los grupos étnicos a la educación superior su apogeo ha sido reciente lo que contrasta con lo escrito por Mato (2012) que "en las últimas dos décadas, la mayoría de las sociedades latinoamericanas han logrado mejorar la calidad de sus democracias respecto de algunas inequidades que afectan particularmente a pueblos indígenas y afrodescendientes (pág. 17).

Cabe destacar lo que plantea González (2011) que los jóvenes pertenecientes a grupos étnicos del país, tienen mucha más educación que las generaciones anteriores. Sin embargo, los niveles educativos alcanzados por los jóvenes todavía no son suficientes en la llamada sociedad de la información y del conocimiento.

\section{CONCLUSIONES}

Para reducir la desigualdad educativa se deben contrarrestar los procesos de exclusión, discriminación y desigualdad que se producen no sólo afuera sino también dentro del sistema educativo hondureño. Aumentar la cobertura sobre todo en las zonas rurales y departamentos más aislados, mejorar la calidad de la educación a través de la capacitación constante a los docentes, mejorar la focalización de los programas educativos para que estos alcancen a los más pobres y en especial que exista una eficiente planificación, seguimiento y evaluación de los planes y programas del sistema educativo, son parte de los aspectos a tomar en cuenta para reducir la desigualdad educativa en el país.

Se deben maximizar los esfuerzos para que todos los grupos y estratos sociales tengan igualdad de oportunidades educativas que permitan, o al menos propicien, la reducción de las brechas sociales y que actualmente se acentúan más en los departamentos pobres del país.

Honduras ha logrado la construcción de una política de educación especial, a sí mismo el desarrollo de un programa intercultural y multilingüe que permite entre otras acciones la inclusión de niños, jóvenes y adultos provenientes de los pueblos indígenas y afro hondureños a todos los procesos de formación académica.

\section{ReFERENCIAS}

Alas, M., \& Moncada, G. (2011). Problemas de Equidad en el Sistema Educativo Hondureño. Revista de Investigación Educativa., 299.

Alianza mundial para la Educación. (2014). 250 millones de razones para invertir en educación: el caso de la Inversión. GPE/Midastouch.

Aponte, A. (2013). Desigualdad, inclusión y equidad en la educación superior en américa latina y el caribe. Ginebra: Eisalc.

Davila, P., Naya, L., \& Asier, L. (2010). Las personas con discapacidad, el derecho a la educación y la convención sobre los derechos del Niño, en America Latina. Revista Latinoamericana de Educación Inclusiva, 117.

Duriez, M., López, V., \& Moncada, G. (2016). Educación superior en Iberoamerica/Informe Nacional Honduras. Tegucigalpa, M.D.C: UNIVERSIA.

Espino, I. (2015). Desigualdad educativa en Honduras: un análisis comparativo por departamento y. Consejo Latinoamericano de Ciencias Sociales (CLACSO), 1-32.

Ferreira, F., Messina, J., Rigoline, J., López, L., Lugo, M., \& Renos, V. (2013). La movilidad económica y el crecimiento de la clase media en américa latina. washington, DC: Creative Commons de Reconocimiento CC BY 3.0.

Flores, M. A. (2012). La investigación de las Ciencias Económicas, Administrativas y Contables en la Educación Superior de Honduras. Economía y Administración (E\&A),, 2(2), 191. Recuperado el 07 de marzo de 2016

González, J. M. (2011). ciudadania juveniel etnica: una aproximacion a la relidad de la jueventid etnica y afrodescendiente de Honduras. united nations debelopment progaram Honduras, 9. 
Mato, D. (2011). Universidades Indigenas de America Latina: Logros, problemas y desafios. Revista Andaluza de Antropologia, 63-85.

Mato, D. (2012). Educacion superior y pueblos indigenas y afrodescendientes en America Latina. educacion superior,pueblos indigenas y afrodescendientes en america latina, 17.

Padilla, R. C. (2011). El crecimiento y desarrollo de la educación superior en Honduras, una perspectiva desde la UNAH. Innovacion Educativa , 89.

Paya, A. (2010). Propuestas realidades y retos del futuro. Inclusión Educativa, 125-142.

Rico, A. (2011). Politicas de Educacióin Educativa en America latina. Educación Inclusiva, 142.

Rodriguez-Montano, G. (2013). Midiendo las desigualdades en educación. Revista de Humanidades y Ciencias Sociales, 157-188.

Solis, M., \& Godoy, G. (2016). Problemas de equidad en el sistema esducativo hondureño. Revista Iberoamericana de Evaluacion Educativa, 3.

Solis, M., \& Moncada, G. (2011). Investigación Educativa en la UPN. INIEES UPNFM, 490

Solis, M., \& Moncada, G. (2016). PROBLEMAS DE EQUIDAD EN EL SISTEMA EDUCATIVO HONDUREÑO. Revista Iberoamericana de Evaluación Educativa, 135-151. 